CrossMark \& click for updates

Cite this: Phys. Chem. Chem. Phys., 2016, 18, 12877

Received 23rd December 2015, Accepted 29th March 2016

DOI: $10.1039 / c 5 c p 07925 j$

www.rsc.org/pccp

\section{A simulation-guided fluorescence correlation spectroscopy tool to investigate the protonation dynamics of cytochrome $c$ oxidase $\dagger$}

\begin{abstract}
A. Wolf $\$$ C. Schneider $\$$ T.-Y. Kim, K. Kirchberg, P. Volz and U. Alexiev*
Fluorescence correlation spectroscopy (FCS) is a single molecule based technique to temporally resolve rate-dependent processes by correlating the fluorescence fluctuations of individual molecules traversing through a confocal volume. In addition, chemical processes like protonation or intersystem crossing can be monitored in the sub-microsecond range. FCS thereby provides an excellent tool for investigations of protonation dynamics in proton pumps like cytochrome $c$ oxidase $(\mathrm{CcO})$. To achieve this, the $\mathrm{pH}$-dependent fluorescent dye fluorescein was attached as a protonation probe to the $\mathrm{CcO}$ surface via site-specific labeling of single reactive cysteines that are located close to the entry point of a proton input channel ( $\mathrm{K}$-pathway). The analysis of protonation dynamics is complicated by overlapping triplet and protonation rates of the fluorophore. A Monte Carlo simulation based algorithm was developed to facilitate discrimination of these temporally overlapping processes thus allowing for improved protonation reaction rate determination. Using this simulation-guided approach we determined precise local proton association and dissociation rates and provide information about protein surface effects, such as proton collecting antennae, on the transport properties of proton transfer channels.
\end{abstract}

\section{Introduction}

As an essential part of the respiratory chain cytochrome $c$ oxidase $(\mathrm{CcO})^{1}$ is present in both eukaryotes and most aerobic prokaryotes. Functioning as a redox-coupled proton pump $\mathrm{CcO}$ physically transports hydrogen ions (protons) through the membrane and chemically uses $\mathrm{H}^{+}$to reduce oxygen to water while oxidizing cytochrome $c$ at the P-side of the membrane. The coupling of this redox-reaction to the translocation of additional protons through the membrane ("proton pumping") increases further the electrochemical trans-membrane proton gradient that is the driving force for ATP synthesis by ATP synthase. However, protonation dynamics, coupling with electron transport and conformational changes associated with $\mathrm{CcO}$ 's function are yet to be fully explained. ${ }^{1-3}$ Scheme 1 a depicts proton pathways in $\mathrm{CcO}$ subunits I and II. To reduce one molecule of oxygen, four electrons are taken up from cytochrome $c$. Cytochrome $c$ which binds to $\mathrm{CcO}$ on the positively charged side (P-side) of the membrane, injects electrons into the bimetallic $\mathrm{Cu}_{\mathrm{A}}$ center, which in turn donates electrons, one at the time,

Department of Physics, Freie Universität Berlin, 14195 Berlin, Germany.

E-mail: ulrike.alexiev@fu-berlin.de

$\dagger$ Electronic supplementary information (ESI) available: Further information on experimental data. See DOI: $10.1039 / \mathrm{c} 5 \mathrm{cp} 07925 \mathrm{j}$

$\$ \mathrm{AW}$ and CS contributed equally to the work.
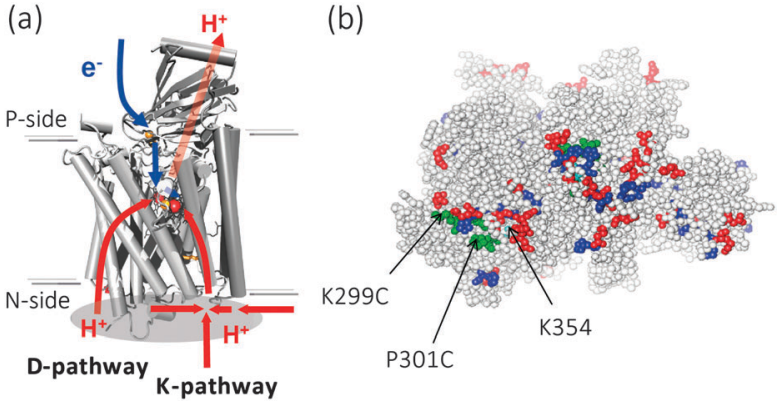

Scheme 1 (a) Scheme of CcO's proton (red) and electron (blue) pathways in the structural model of $\mathrm{CCO}$ (PDB: 3HB3). Coppers $\mathrm{Cu}_{A} / \mathrm{Cu}_{B}$ (orange spheres) and hemes $a / a_{3}$ (stick representation with red Fe spheres) are indicated. (b) Top view onto the cytoplasmic surface. Acidic amino acids are colored red and histidines blue. Residues exchanged with cysteines are highlighted in green. $\mathrm{K} 299 \mathrm{C}$ and P301C, close to the entrance of the $\mathrm{K}$ channel are indicated. K354 is shown in cyan.

to heme $a$ (Scheme 1a). From there electrons are passed on to the heme $a_{3} / \mathrm{Cu}_{\mathrm{B}}$ binuclear site. Molecular oxygen is supposed to bind to the heme $a_{3}$ iron when both the heme iron and $\mathrm{Cu}_{\mathrm{B}}$ are reduced after transfer of the second electron. ${ }^{1-3}$

Two separate proton pathways have been suggested for the bacterial $\mathrm{C} c \mathrm{O}$, leading from the $\mathrm{N}$-(negative) side (cytoplasmic side) of the membrane towards the heme-cooper site: the so-called D- and K-pathways ${ }^{4,5}$ (Scheme 1a). 
Recent results on proton uptake and release during the $\mathrm{O} \rightarrow \mathrm{E}$ step in the catalytic cycle ${ }^{6}$ provided evidence for the existence of a proton collecting antenna in $\mathrm{CcO},{ }^{7}$ switched on by electron injection. ${ }^{6}$ Proton collecting antennae to concentrate protons at the membrane surface and near the entry point of a proton-input channel were initially suggested for proton uptake by bacteriorhodopsin ${ }^{8}$ and are also discussed for the Green Fluorescent Protein (GFP). ${ }^{9}$ This is connected to the idea of localized proton circuits along the membrane, facilitating proton transfer from a proton pump to a proton sink (e.g. ATP synthase). A similar electrostatics based mechanism of a transient proton collecting antenna as observed for $\mathrm{CcO}$ might occur in bacteriorhodopsin where proton uptake and its sequence in the photocycle correlated with a transient increase in negative surface charge. ${ }^{10,11}$

Observation of fast lateral proton transfer along membrane surfaces $^{12-15}$ support the idea of proton collecting antennae. Estimates of proton diffusion constants at the membrane surface that may even be larger than the diffusion constant of protons in bulk water were derived from protonation changes of fluorescein bound to the surface of bacteriorhodopsin membranes ${ }^{13}$ and are supported by recent measurements on lipid membranes. ${ }^{16}$ Further, measurements of apparent protonation second-order rate constants at the protein or membrane surface that exceed those corresponding to proton diffusion in aqueous solution $\left((1-6) \times 10^{10} \mathrm{M}^{-1} \mathrm{~s}^{-1}\right),{ }^{6,12,17,18}$ also in a $\mathrm{pH}$ dependent fashion, ${ }^{19,20}$ were reported. Together these observations suggest that the rate of proton supply is modified by the properties of the protein surface and the surrounding membrane. This raises the following question: What is the exact pathway which is taken by the proton from the bulk to the surface, along the surface, from the surface to the channel and through the channel until it is released on the other side of the membrane (see Scheme 1a)?

Fluorescence correlation spectroscopy ${ }^{21}$ (FCS) is particularly useful to investigate these questions as it allows to directly obtain the apparent protonation exchange rates of a single fluorophore. $^{20}$ The key observable in FCS is the intensity fluctuation in the fluorescence signal. ${ }^{22}$ Chemical reactions can be monitored via FCS when the fluorescence intensity is sensitive to the substrate, i.e. in our case to the proton. The fluorescence autocorrelation will be affected and the kinetic parameters of the underlying photophysical process of the fluorophore will be accessible. In particular, FCS results on lateral proton transfer at lipid membrane surfaces ${ }^{20}$ and between the membrane and a membrane protein ${ }^{17}$ showed an enhancement of proton association and suggested that at higher $\mathrm{pH}$ values the whole lipid membrane would act as a proton collecting antenna. ${ }^{20}$ Another example is the protonation reaction of EGFP, a $\mathrm{pH}$ dependent fluorescent protein. ${ }^{23}$ The different kinetic parameters of the EGFP protonation reaction were extracted from the $\mathrm{pH}$ dependent fluorescence autocorrelation functions.

In this work we focus on the effect of the protein surface on the protonation dynamics. Using site-directed fluorescence labeling (SDFL) of single-cysteine $\mathrm{CcO}$ variants ${ }^{24}$ we aim in the long run at mapping the protein surface and the entrance of the proton pathways with the protonation reporter dye fluorescein (Scheme 1b-d). Fluorescein is a fluorescent dye with a strong $\mathrm{pH}$ dependent absorption band with a $\mathrm{p} K$ of around 6.4 for the transition of the monoanionic to the alkaline dianionic form. ${ }^{25}$

To overcome a known challenge in extracting protonation rates from FCS data, we report here a simulation-guided analysis that allows us to discriminate between overlapping triplet and protonation rates, resulting in an improved reaction rate determination. Precise knowledge of these rates is mandatory to unambiguously demonstrate the existence of proton collecting antennae and their constituents. The procedure was tested on fluorescein free in solution and bound at specific positions on the surface of $\mathrm{CcO}$ from $P$. denitrificans. Protonation on $\left(k_{+1}=(9.5 \pm 0.7) \times 10^{10} \mathrm{M}^{-1} \mathrm{~s}^{-1}\right)$ and off $\left(k_{-1}=(2.7 \pm 0.3) \times 10^{4} \mathrm{~s}^{-1}\right)$ rates in the vicinity of the entry to the K-channel show slightly increased proton association rates in the oxidized state of the enzyme directly at the protein surface.

\section{Experimental section}

\subsection{Materials}

$n$-Dodecyl- $\beta$-D-maltoside (DM) was from GLYCON Biochemicals GmbH (Luckenwalde, Germany). 5-Iodoacetamidofluorescein (5-IAF) was from Invitrogen/Molecular Probes. All other chemicals were of the highest grade available.

\subsection{Sample preparation}

Preparation of cysteine variants from $P$. denitrificans was performed as described. ${ }^{26}$ Briefly, as a background mutant the CS (C140S/C48S) variant was used. Essential cysteines, such as $\mathrm{C} 216$ and $\mathrm{C} 220$ which serve as ligands for $\mathrm{Cu}_{\mathrm{A}}$, and the pair C66/C80 which forms a disulfide bridge as well as cysteines in the protein core (in position 50 and 56 of subunit II) were not changed. Cells were grown on succinate medium, ${ }^{27}$ supplemented with $25 \mu \mathrm{g} \mathrm{ml}{ }^{-1}$ streptomycin sulfate and $25 \mu \mathrm{g} \mathrm{ml}$ kanamycin at $32{ }^{\circ} \mathrm{C}$. Enzyme preparation of core subunits I and II and variants was performed as described, ${ }^{26}$ followed by the addition of $0.1 \%$ LDAO to detach the core subunits from subunit III and IV. Separation and detergent exchange was done on a Q-Sepharose FF ion exchange column. For complete reduction of $\mathrm{CcO} 2 \mathrm{mM}$ sodium dithionite was added to the solution. The $\mathrm{CcO}$ concentration was determined from the reduced minus oxidized optical difference spectrum with $\varepsilon_{605-630 \mathrm{~nm}}=11700 \mathrm{M}^{-1} \mathrm{~cm}^{-1} \cdot{ }^{28}$

\subsection{Determination of enzymatic activities of isolated $\mathrm{CcO}$}

Cytochrome $c$ oxidase activity was determined as described previously. ${ }^{29}$ Reduced horse heart cytochrome $c$ was used at a concentration of $50 \mu \mathrm{M}$ in $10 \mathrm{mM}$ potassium phosphate buffer $\mathrm{pH}$ 7.4, $40 \mathrm{mM} \mathrm{KCl,} 1 \mathrm{mM}$ EDTA and $0.05 \% \mathrm{DM}$ at room temperature. The rate of cytochrome $c$ oxidation was measured by following the change in absorbance at $550 \mathrm{~nm}$. 


\subsection{Labeling with 5-iodoacteamidofluorescein}

5-IAF is a $\mathrm{pH}$ dependent fluorescein derivative that readily reacts with the thiol group of cysteines. The $\mathrm{p} K$ of the transition to the alkaline form is 6.2 in the unbound state. ${ }^{12,30}$ For the labeling of $\mathrm{CcO}$ with IAF to a solution of $50 \mu \mathrm{M} \mathrm{C} c \mathrm{O}$ in $10 \mathrm{mM}$ potassium phosphate buffer $\mathrm{pH} 7.5,50 \mathrm{mM} \mathrm{KCl}$ and $0.05 \% \mathrm{DM}$ a tenfold molar excess of 5-IAF over $\mathrm{CcO}$ was added. The mixture was allowed to react for $1 \mathrm{~h}$ at room temperature in the dark. Unbound label was removed via gel filtration. The labeling stoichiometry was calculated spectroscopically from the absorbance at $425 \mathrm{~nm}$ of $\mathrm{CcO}$ and the absorbance change of fluorescein at $495 \mathrm{~nm}$ :

$$
\frac{[\mathrm{F}]}{[\mathrm{Cc} \mathrm{O}]}=\frac{\Delta A_{495}}{\Delta A_{425}} \frac{\varepsilon_{425}^{\mathrm{C} c \mathrm{O}}}{\Delta \varepsilon_{495}^{\mathrm{F}}}
$$

with

$$
\Delta A_{495}=\Delta A_{495}^{\mathrm{pH} 9.5}-\Delta A_{495}^{\mathrm{pH} 5.5}
$$

The extinction coefficients are $\Delta \varepsilon_{495}=36000 \mathrm{M}^{-1} \mathrm{~cm}^{-1}$ (calculated from the value for the alkaline form of fluorescein given by Molecular Probes) and $\varepsilon_{425 \mathrm{~nm}}=158000 \mathrm{M}^{-1} \mathrm{~cm}^{-1}$ for $\mathrm{CcO}$. Covalent binding of IAF was checked via SDS polyacrylamide gel electrophoresis. The fluorescein labeled $\mathrm{C} c \mathrm{O}$ variants (CcO-AF) exhibit a labeling stoichiometry of $26 \%$ for CS-K299C-AF, and $75 \%$ for CS-P301C-AF. ${ }^{24}$ After labeling the enzymatic activity was checked. ${ }^{24}$

\subsection{UV/Vis and fluorescence spectroscopy}

$\mathrm{UV} /$ Vis absorption spectra were measured at room temperature (19-20 $\left.{ }^{\circ} \mathrm{C}\right)$ using a standard double-beam UV/VIS absorption spectrophotometer UV-2450PC from Shimadzu and $3 \times 3 \mathrm{~mm}$ quartz cuvettes.

\subsection{FCS measurements}

Scheme 2 shows the measurement setup and measurement principle. The sample was diluted to appropriate concentrations $(\sim 2 \mathrm{nM})$ in $100 \mathrm{mM} \mathrm{KCl}, 0.05 \% \mathrm{DM}$ and purged with nitrogen to remove traces of $\mathrm{CO}_{2} \cdot \mathrm{pH}$ was measured throughout the measurements. FCS measurements were performed on a confocal setup, consisting of an Olympus IX70 microscope, a linear polarized Argon ion laser (Melles Griot series 543, Melles Griot, Carlsbad, CA) operated at $488 \mathrm{~nm}$ and a confocal scanning system DSC-120 (Becker\&Hickl, Berlin, Germany). The laser was coupled into the microscope via the DSC-120 system. The laser beam was then focused by a $60 \times$, NA 1.2, UPlanApo Olympus water immersion objective into the sample. Sample fluorescence was collected through the same objective and passes a telescope before being imaged onto a pinhole $(0.5 \mathrm{~mm})$. After the pinhole, the emission beam passes two filters for blocking the laser light and selecting the emission wavelength range (long-pass LP515 and band-pass HQ525/50 (AHF Analysentechnik GmbH, Tübingen, Germany)) before hitting the GaAsP hybrid detector (bH-HPM-100-40, Becker\&Hickl, Berlin, Germany). ${ }^{31}$ The signal was processed by a DPC230 photon correlator card (Becker\&Hickl, Berlin, Germany).
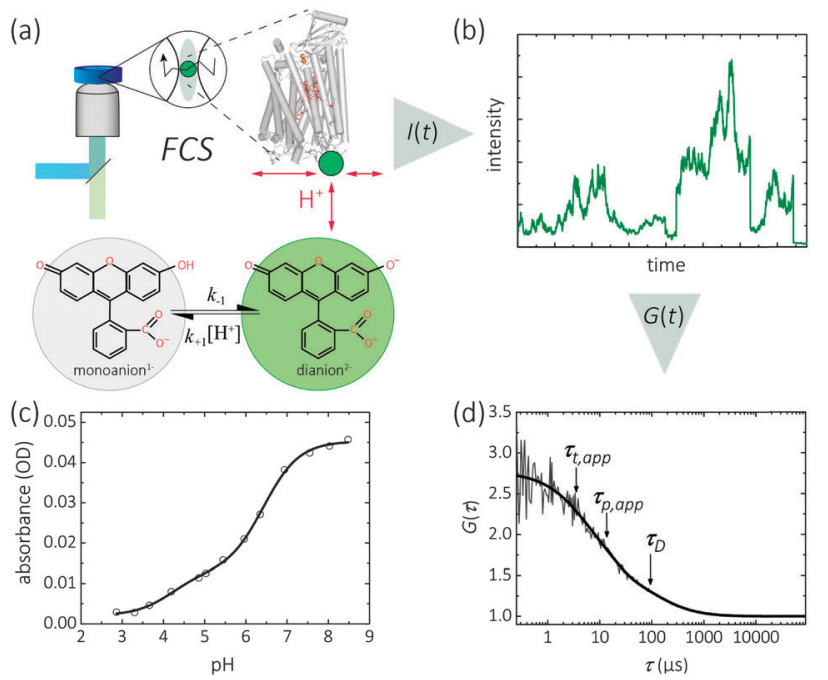

Scheme 2 (a) Schematic of the experimental FCS setup. The fluoresceinlabeled cytochrome $c$ oxidase freely diffuses in solution. It's traversing through the excitation volume while in a fluorescent state (dianion ${ }^{2-}$ ) in contrast to non-fluorescent states (monoanion ${ }^{1-}$ /triplet) results in fluorescence being picked up by a detector which measures (b) the fluctuating fluorescence intensity over time. (c) The $\mathrm{pH}$ titration curve for 5-IAF shows decreasing absorbance for lower values of $\mathrm{pH}$ corresponding to lower molecular extinction coefficients of the increasingly protonated forms (monoanion, neutral) of fluorescein. (d) From the intensity fluctuations the autocorrelation is calculated and exhibits distinctive decay components with characteristic times $\tau$ for diffusion through the excitation volume $\tau_{\mathrm{D}}$, protonation-deprotonation $\tau_{\mathrm{p} \text {,app }}$ and singlet-triplet transitions $\tau_{\text {t,app. }}$

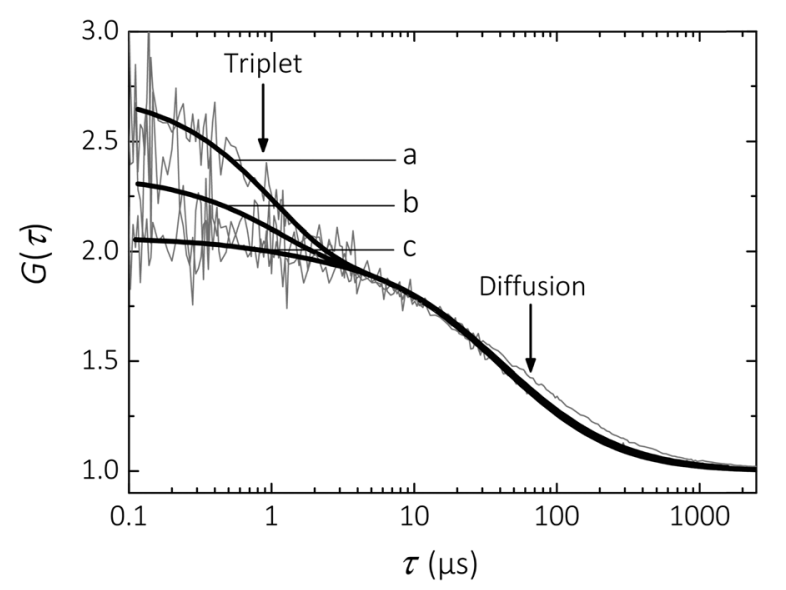

Fig. 1 Autocorrelation curves of fluorescein in $0.1 \mathrm{M} \mathrm{NaOH}$ at three different laser intensities $(1 \mu \mathrm{W}, 2 \mu \mathrm{W}, 20 \mu \mathrm{W})$. The power was reduced from (a) to (c). The contribution of molecules residing in the triplet state to the FCS curves reduces with reducing laser power. Under the conditions in (c) only a minor contribution in the $1 \mu$ s time range was observed.

Before starting the fluorescein titration experiments, the laser intensity was tested and reduced (from about $100 \mu \mathrm{W}$ to $1 \mu \mathrm{W}$ ) until the contribution of the triplet state to the fluorescein FCS curve was negligible (Fig. 1) and an autocorrelation curve with a sufficient signal-to-noise ratio could be measured. The laser intensities used are given in the respective figure legends. 


\subsection{Analysis of FCS curves}

The recorded correlation curves were analyzed using nonlinear least-squares Levenberg-Marquardt fitting routines. The autocorrelation of the fluorescence intensity is calculated by:

$$
G(\tau)=\frac{\langle F(0) F(\tau)\rangle}{\langle F(\tau)\rangle^{2}}
$$

The angular brackets indicate the time average, $F$ is the fluorescence signal as a function of time and $\tau$ is the lag time. The detection volume is assumed to be a three-dimensional Gaussian, whose shape is described by the parameters $\omega_{x y}$ (horizontal axis) and $\omega_{z}$ (perpendicular axis). ${ }^{32}$ The following equation (adapted from ref. 17 and 20) was used to fit the normalized autocorrelation function:

$$
\begin{aligned}
& G(\tau)= \\
& 1+\frac{1-A_{\text {prot }}-A_{\text {trip }}+A_{\text {prot }} \times \exp \left(-\frac{\tau}{\tau_{\text {p,app }}}\right)+A_{\text {trip }} \times \exp \left(-\frac{\tau}{\tau_{\text {t,app }}}\right)}{N\left(1-A_{\text {prot }}-A_{\text {trip }}\right)\left(1+\frac{\tau}{\tau_{\mathrm{D}}}\right)\left(1+\frac{\tau}{\tau_{\mathrm{D}}}\left(\frac{\omega_{z}}{\omega_{x y}}\right)^{2}\right)^{1 / 2}}
\end{aligned}
$$

with $N$ the average number of fluorophores in the detection volume, $A_{\text {prot }}$ the relative fraction of molecules residing in the protonated state, and $A_{\text {trip }}$ the relative fraction of molecules residing in the triplet state. The fluctuations due to protonation and deprotonation of fluorescein give rise to a correlation relaxation time $\tau_{\mathrm{p} \text {,app }}$, the respective correlation time for the triplet-singlet relaxation is $\tau_{\mathrm{t} \text {,app }}$ and $1 / \tau_{\mathrm{p} \text {,app }}$ and $1 / \tau_{\mathrm{t} \text {,app }}$ are the apparent rate constants for protonation and triplet events, respectively. $\tau_{\mathrm{D}}$ denotes the diffusion time of the molecule.

Eqn (4) can also be expressed in the following way where the individual terms of protonation and triplet events are more easily recognizable:

$$
\begin{aligned}
G(\tau)= & 1+g_{0} \frac{1}{\left(1+\frac{\tau}{\tau_{\mathrm{D}}}\right) \sqrt{1+\frac{\tau}{\tau_{\mathrm{D}}\left(\frac{\omega_{z}}{\omega_{x y}}\right)^{2}}}} \\
& \times\left(1+\frac{A_{\text {prot }}}{1-A_{\text {prot }}-A_{\text {trip }}} \mathrm{e}^{-\frac{\tau}{\tau_{\text {papp }}}}+\frac{A_{\text {trip }}}{1-A_{\text {prot }}-A_{\text {trip }}} \mathrm{e}^{-\frac{\tau}{\tau_{\text {t,app }}}}\right)
\end{aligned}
$$

with $g_{0}=\gamma / N$. For convenience the shape-factor $\gamma$ was consistently assumed to be 1 (therefore $g_{0}{ }^{-1}=N$ ) and the effective volume accordingly calculated to be $V_{\text {eff }}=\pi^{3 / 2} \omega_{x y}{ }^{2} \omega_{z}$. For a detailed discussion of the relation between the shape-factor and concentration in the effective volume see ref. 33. In the formulation of eqn (5) one can easily see that both the protonation as well as the triplet term are treated identical in the fitting of the FCS data. The factor before the exponential terms is the time the particle spends in the dark state due to the respective process (protonation/ISC) in relation to the time the particle spends in the overall light state. However, the apparent time constants $\tau_{\mathrm{p} \text {,app }}$ and $\tau_{\mathrm{t} \text {,app }}$ are dependent on both the on- and off-rates of the respective processes, and the inter-system crossing depends on the excitation energy, which is nonhomogeneously distributed over the excitation volume. Thus, it is not sufficient to just discriminate the time constants by assuming the shorter time to be caused by inter-system crossing.

\subsection{Monte Carlo simulation for generating FCS data}

All Monte Carlo simulations were run as a self-written script in Mathematica Version 9. The program employs a number of independent fluorophores diffusing (random walk) inside a box cell with an enclosed confocal excitation/emission volume thus exhibiting diffusion-induced fluorescence fluctuation measured by a "detector" while also employing dynamic processes such as protonation-dependent changes in quantum yield and inter-system crossing (ISC). The following parameters were set as input values for the simulation: the mean concentration $N$ of particles in the effective excitation volume $V_{\text {eff }}$ $(N=1)$, the size of the cubic box cell with continuous boundary conditions in which the particles of the simulations diffuse (axis $a=100$ length units), the diffusion constant of free fluorescein $\left(D=0.04 \mu \mathrm{m}^{2} \mathrm{~s}^{-1}\right),{ }^{34}$ the number of iterations of the overall simulation (10), the number of frames in each simulation (10 million), the axes of the confocal Gaussian ellipsoid ( $\omega_{z}=3 \times \omega_{x y}=30$ units), and the time resolution of the simulation i.e. the temporal distance between two consecutive frames $(\delta t=10 \mathrm{~ns})$. Additionally macroscopic amplitudes for ISC and protonation events $\left(A_{\text {prot }}, A_{\text {trip }}\right)$ can be set between 0 and 1.

Using these parameters the algorithm calculates the effective volume $V_{\text {eff }}$ of the Gaussian ellipsoid. The pixel resolution is then set to $\delta x=2^{-1 / 2} \times\left(10^{-12} / V_{\text {eff }}\right)^{1 / 3}$. The diffusivity generating the random movement of particles in the box cell then follows as time resolution $\delta t /(\delta x)^{2}$. Also established is the number of particles $\mathrm{n}$ needed in the box cell to achieve the concentration $N$ of particles in the confocal volume as $n \approx N \times a^{3} / V_{\text {eff }}$.

For time-dependent fluorescence fluctuation processes not resulting from the diffusion of fluorophores but from the quantum chemical characteristics of $\mathrm{pH}$-dependent fluorophores, the algorithm also emulates on-off-state transitions for either a protonation induced quantum yield drop as observed for fluorescein, or inter-system crossing, a common property of all fluorescent dyes. The characteristic macroscopic parameters emerging from these effects in a fluorescence correlation spectroscopy experiment are the amplitudes $A_{\text {prot }}$ and $A_{\text {trip }}$ of both processes ranging from 0 to 1 , where a value of 0 corresponds to no observed fluctuation and increasing values yield the average percentage of time all fluorophores stay in the off-state. The second observed macroscopic parameter is the expected lifetime of the off-states $\tau_{\text {prot }}$ and $\tau_{\text {trip. }}$. In the offstate of a particle the individual quantum yield is set to 0 and no fluorescence from that particle reaches the "detector".

The expected rates for a transition into the off-state are calculated as:

$$
k_{\text {prot }}^{+1}=\frac{A_{\text {prot }}}{\tau_{\text {prot }}\left(1-A_{\text {prot }}\right)},
$$




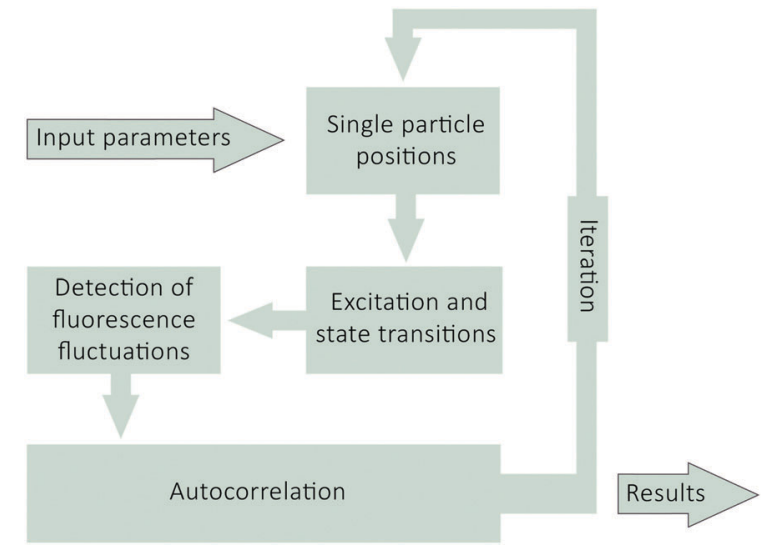

Scheme 3 Schematic outline of the single-molecule based simulation of fluorescence correlation spectroscopy data.

and

$$
k_{\text {trip }}^{+1}=\frac{A_{\text {trip }}}{\tau_{\text {trip }}\left(1-A_{\text {trip }}\right)} \text {. }
$$

For protonation induced off-states the expected probability for a particle is uniformly distributed over the entire box cell. For triplet transitions, however, there is a factor $m$ needed in order to scale the local excitation intensity dependent transition probability up to the expected probability when accounting for the distribution intensity $I(r)$ in the box cell. The microscopic off-transition probability for a particle at position $p$ is then given by $m \times I(p)$. The factor $m$ is determined numerically by calculating the average intensity $\langle I(r)\rangle$ in the box cell. From the average intensity, $m$ then follows as $m=k_{\text {trip }}^{+1} /\langle I(r)\rangle$. At the beginning of the simulation for each particle a pseudorandom starting position is determined, which is uniformly distributed in the box cell. The movement of each particle from its position $r_{i}$ in frame $i$ to $r_{i+1}$ in the consecutive frame is generated from a pseudorandom Gaussian probability distribution with expected value 0 and standard deviation $\sqrt{2 D \times \delta t}$. After the generation of the entire movement of all particles for each frame a pseudorandom real number in the range $[0,1]$ is generated, either for a protonation event induced off-state or for a singlettriplet transition. If this number is smaller than the microscopic transition probability, the particle undergoes the transition and stops fluorescing for a time generated from an exponential probability distribution with characteristic time $\tau_{\text {prot }}$ or $\tau_{\text {trip. }}$. In the off-state the "detector" will not pick up a signal from that particle. The off-state duration generated from the exponential probability is a real number. Therefore, as a particle starts to emit again in the middle of a frame only a partial fluorescence is generated. As the simulation runs in discrete time steps the start of the off-state was generated from a uniform distribution with range $[-1,1]$ and a partial fluorescence was subtracted from the starting frame. To obtain the intensity time trace of the particle ensemble, the individual particle intensities were added together and the autocorrelation was calculated according to Section 1 . The simulation is repeated depending on the number of iterations chosen and the individual autocorrelations were subsequently averaged. Scheme 3 outlines the simulation algorithm. The resulting FCS curves were fitted with eqn (4).

\section{Results and discussion}

\subsection{Measurement of proton association-dissociation rates at position 301 of $\mathrm{CcO}$}

Fluorescence fluctuations of fluorescein covalently bound to position 301 close to the K-channel were evaluated as a function of pH. Fig. 2a shows the correlation curves of fluorescein at two selected $\mathrm{pH}$-values. The slow relaxation time corresponds to the average diffusion time $\left(\tau_{\mathrm{D}}\right)$ of the labelled $\mathrm{CcO}$ in detergent solution through the detection volume. Another relaxation becomes visible in the time range that lies between the relaxation time due to singlet-triplet transitions of the fluorophore $\left(\tau_{\mathrm{t}, \text { app }}\right.$ in Fig. 1) and the diffusion time (Fig. 2b). This relaxation component is $\mathrm{pH}$ dependent and describes the fluorescence
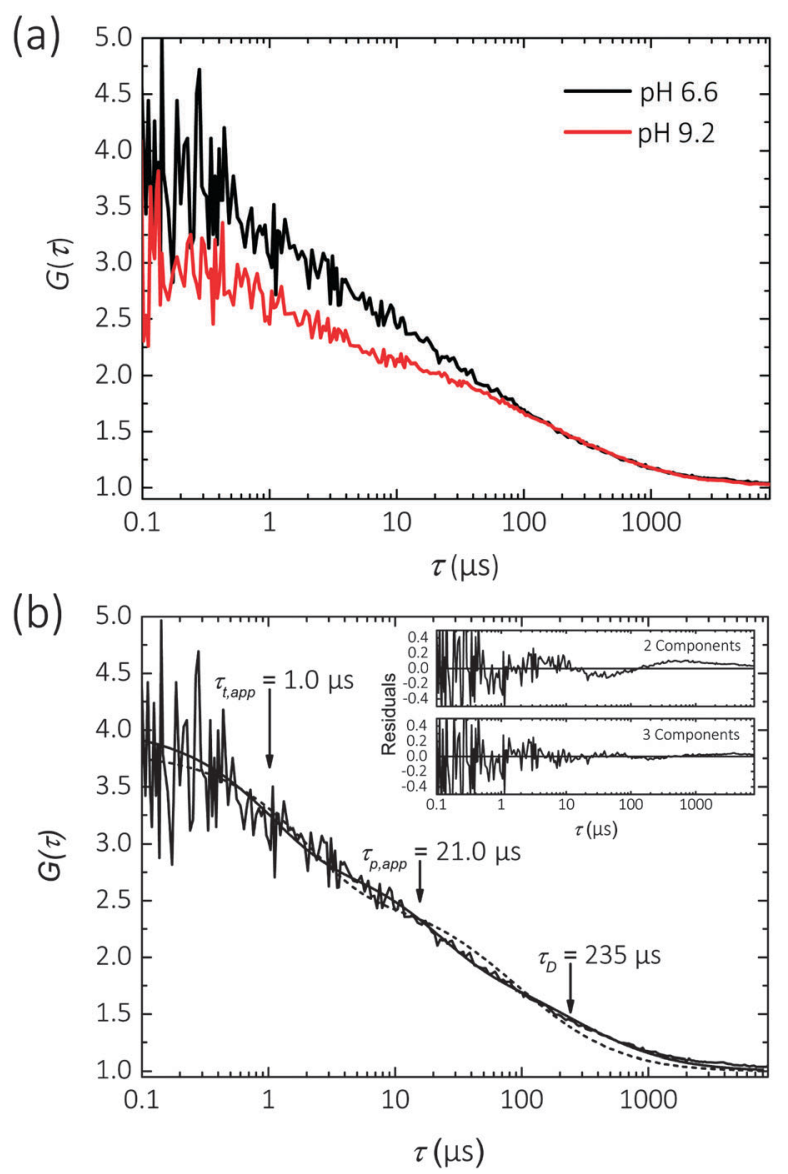

Fig. 2 Autocorrelation curves of $\mathrm{CcO}$ CS-P301C-AF at selected $\mathrm{pH}$ values. (a) Comparison between $\mathrm{pH}$ values 6.6 and 9.2 with the curves normalized to $g_{0}{ }^{-1}=1$. (b) Fit of the autocorrelation curve at $\mathrm{pH} 6.6$ with eqn (4). Fit curves with two (dashed line) and three (straight line) components are shown. A fit with three components as indicated by the arrows and the corresponding time constants describes the data sufficiently. Inset: The corresponding fit residuals are presented. Sample conditions: $100 \mathrm{mM} \mathrm{KCl}, 0.05 \% \mathrm{DM}, 21^{\circ} \mathrm{C}$. Excitation as described in the Experimental section. Excitation intensity: $5 \mu \mathrm{W}$. 


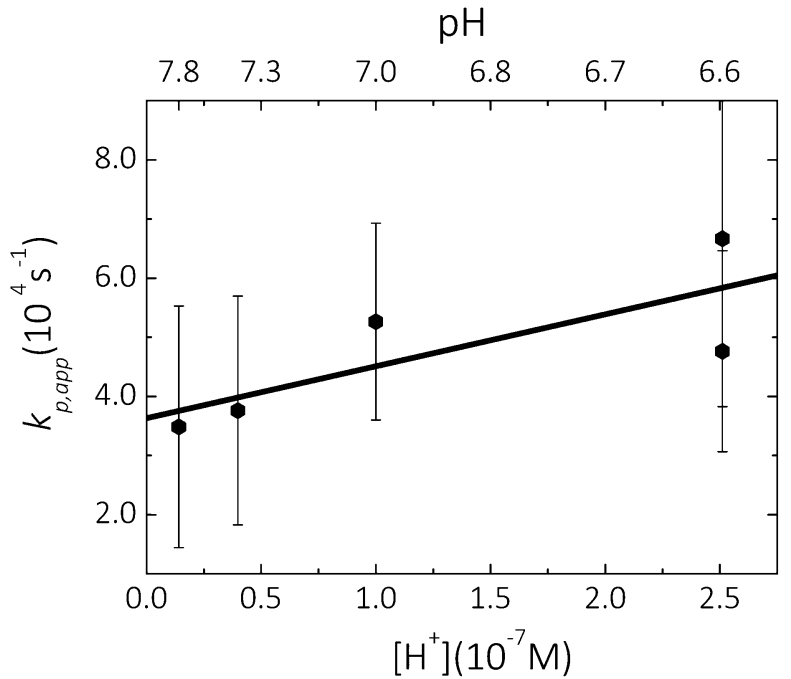

Fig. 3 Protonation rate $k_{\text {p,app }}$ as a function of proton concentration for CCO CS-P301C-AF. The data were fitted to eqn (7). Conditions as in Fig. 2.

intensity fluctuations due to protonation and deprotonation of the bound fluorescein with the correlation time $\tau_{\mathrm{p} \text {,app }}$, (Fig. 2a and b).

The corresponding rate constant (see also ESI $\dagger$ ), $k_{\mathrm{p}, \text { app }}=$ $1 / \tau_{\mathrm{p} \text {,app }}$, was plotted as a function of $\mathrm{pH}$ in Fig. 3 . In the $\mathrm{pH}$ range between 6.5 and 8 a linear dependence between protonation rate and proton concentration was observed for CS-P301C-AF. The following on $\left(k_{+1}\right)$ and off $\left(k_{-1}\right)$ rates were determined from the linear fit to the model:

$$
k_{\mathrm{p}, \text { app }}=k_{+1}\left[\mathrm{H}^{+}\right]+k_{-1}
$$

The protonation on and off rates extracted from eqn (7) were $k_{+1}=(9 \pm 4) \times 10^{10} \mathrm{M}^{-1} \mathrm{~s}^{-1}$ and $k_{-1}=(3.6 \pm 0.6) \times 10^{4} \mathrm{~s}^{-1}$, respectively. The $\mathrm{p} K_{\mathrm{a}}$ value as determined from the logarithm of the ratio of the on and off rates is 6.4, close to the value of IAF in solution with $\mathrm{p} K_{\mathrm{a}}=6.2,{ }^{35}$ and indicates only a slight effect of a negative surface potential. Similar results were obtained by previous pH-dependent absorption measurements close to the K-channel. ${ }^{24}$

\subsection{Dependence of the shape of the autocorrelation curve and the corresponding $g_{0}$-value on the chemical reaction leading to fluorescence fluctuations}

The fundamental difference between protonation and ISC induced fluorescence fluctuations is the way the underlying chemical transitions occur in the FCS experiment. Since protons are homogeneously distributed in the solution the probability of protonation/deprotonation reactions is uniform in the system i.e. there is no local correlation. For ISC, however, this holds not true in a confocal fluorescence excitation geometry. The higher the excitation intensity the larger is the chance that a fluorophore crosses into a long-lived triplet state (inter-system crossing). Due to this dependence on the excitation light intensity, which is inhomogeneous and highest at the center of the Gaussian excitation ellipsoid, the ISC probability is locally correlated. This important distinguishing property between the two time-dependent fluctuation processes (protonation and ISC) is not explicitly treated in the functions generally used to fit FCS data (eqn (4)), and the protonation and ISC amplitudes are treated equally. However, the difference between the two fluctuation inducing processes is implicitly included in the $g_{0}$ factor. The value for $g_{0}$ (eqn (5)) is inversely connected to the number of fluorophores $N$ in the confocal volume, which at higher intensities alters it's shape due to the reduced number of emitted photons from the remaining fluorophores in the effective volume that are not in the triplet state. This gives rise to a apparent increase in the $g_{0}{ }^{-1}$-value, which in this sense is a shape-concentration factor (see Experimental section and ref. 33 and 36). Fig. 4 demonstrates this result based on the simulated FCS curves and a subsequent fit to eqn (4). In the FCS curve in Fig. 4 a the virtual rise in concentration becomes apparent as a decrease of the intercept in the diffusion part of the autocorrelation function for $\tau \rightarrow 0$ from 2 to 1.55 (visualized as dashed line for the triplet amplitude of 0.52 in Fig. 4a). Fig. $4 \mathrm{c}$ shows the discussed increase in the $g_{0}{ }^{-1}$-value as a function of the amplitude of the singlet-triplet reaction. For protonation events, the fast protonation part and the slower diffusion part of the FCS curve are additive, thus no change in the diffusion part's intercept with the $G(\tau)$-axis is observed (Fig. $4 \mathrm{~b}$ ) and the contribution to the $g_{0}{ }^{-1}$-value remains constant, as can be seen in Fig. 4c. This means that $g_{0}$ is a function of $A_{\text {trip }}$ but not of $A_{\text {prot }}$.

For fluorescent dyes the change in triplet amplitudes is not necessary a result of the intensity of the laser beam, but can also be induced by other molecular effects that are e.g. proton concentration related. This means that in titration experiments different amounts of ISC and therefore an increase in $g_{0}{ }^{-1}$ independent of excitation intensity can occur at different $\mathrm{pH}$ values. This is in contrast to the assumption that the contribution to the triplet part remains constant and only the protonation part of the FCS curve is $\mathrm{pH}$ dependent in correlation time and amplitude. ${ }^{17,20,37}$ As both protonation and triplet processes usually appear in the same time range, the above discussed scenario complicates the analysis of the FCS autocorrelation curves and induces additional uncertainties in the extracted parameters. We now suggest that the $g_{0}$-value can be used to distinguish protonation and photo-induced (e.g. inter-system crossing) processes.

\section{3 $\mathrm{pH}$ dependence of experimental FCS curves}

Using the approach outlined above, we analyzed the FCS curves of fluorescein, IAF and fluorescein bound to the surface of $\mathrm{CcO}$ (see also ESI $\dagger$ ). We observed a rise in the absolute $g_{0}{ }^{-1}$ values of the non-normalized FCS curves of fluorescein at $\mathrm{pH}$ values below 7 (Fig. 5a). As demonstrated in Section 3.2 this rise may stem from a photo-induced process (Fig. 4a and c). When analyzed accordingly (Fig. 5b), we obtain for the experimental case at hand a similar dependence between $g_{0}{ }^{-1}$ and amplitude values as in the simulation of increasing triplet contribution (Fig. 4a and 5b). The inset in Fig. 5b also shows the rise in photo-induced amplitude to be correlated with the formation of the monoanionic species of fluorescein. This rise may be due to increased photoionization or the result of an increased triplet quantum yield for the monoanionic species of fluorescein. 

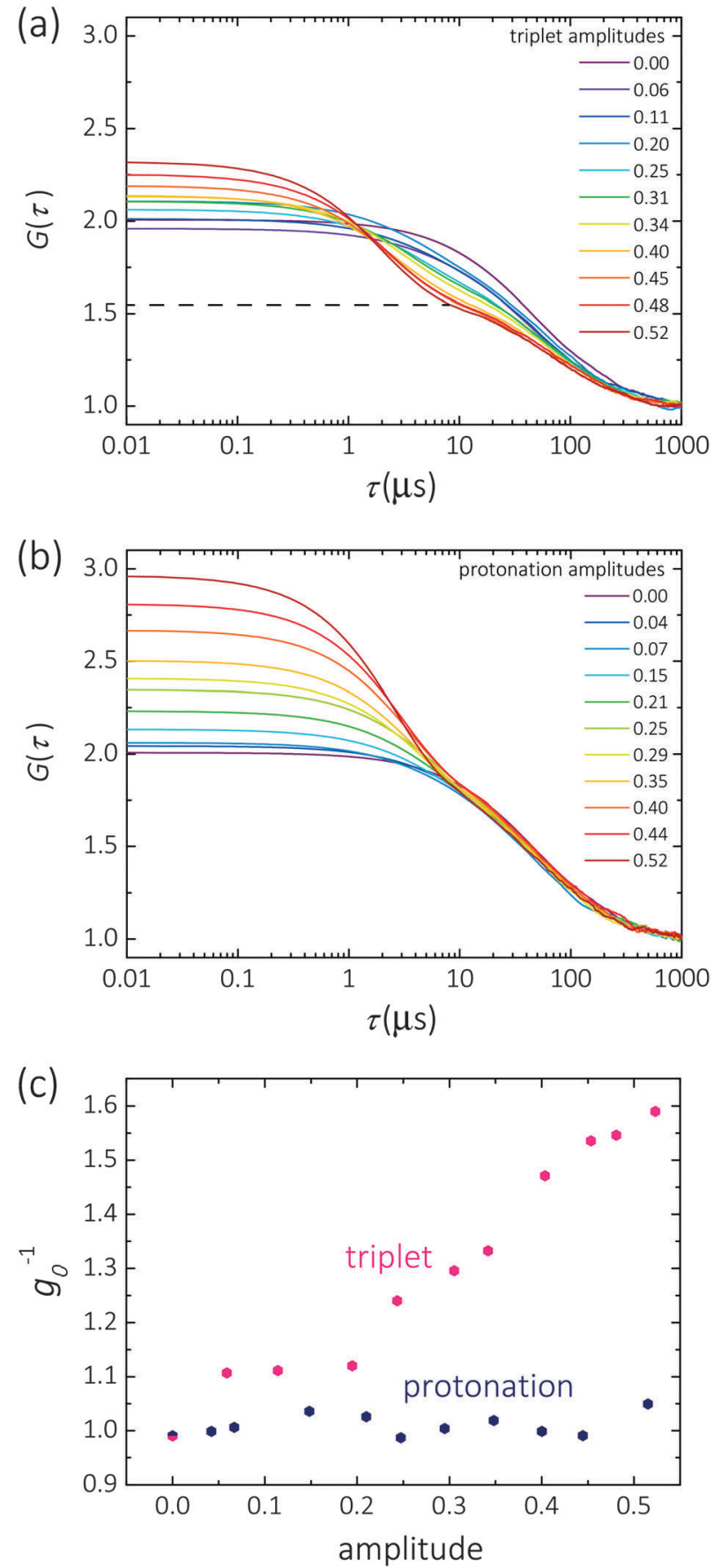

Fig. 4 Autocorrelation curves from simulated data. (a) For increasing triplet amplitudes an additional exponential with amplitude $A_{\text {trip }}$ is added onto the diffusion correlation curve while decreasing it's amplitude. (b) For protonation induced changes in quantum yield only an additional exponential with amplitude $A_{\text {prot }}$ is added onto the diffusion correlation curve, leaving the protonation-subtracted intercept of the diffusion correlation unchanged. (c) Increase of $g_{0}{ }^{-1}$ with higher ISC-triplet amplitudes (pink) and no change in $g_{0}{ }^{-1}$ for all the tested protonation amplitudes (blue), as obtained from fitting of the simulated data in panel (a) and (b) to eqn (4).

Interestingly, we observed this behavior both for fluorescein free in solution and covalently bound at the surface of $\mathrm{CcO}$.
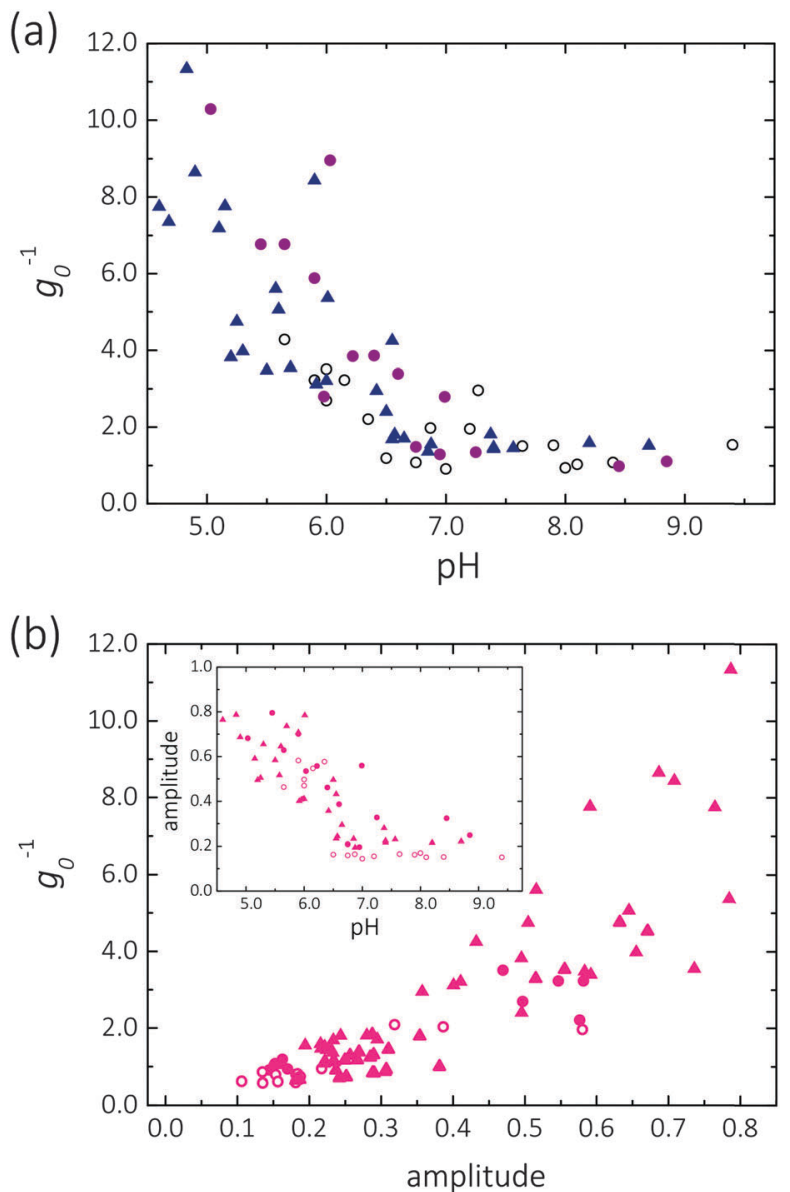

Fig. 5 (a) Fit values for $g_{0}{ }^{-1}$ of fluorescein sodium salt (open circle), 5-iodacetamido-fluorescein (filled circle) as well as fluorescein bound to $\mathrm{CcO}$ from $P$. denitrificans in the oxidized state of the enzyme (triangle). Below $\mathrm{pH} 7$ a clear rise in $g_{0}{ }^{-1}$ is observable for all 4 samples. (b) $g_{0}{ }^{-1}$-values as a function of ICS-triplet amplitudes of the three samples. Inset: Triplet amplitude dependence on $\mathrm{pH}$. Sample conditions: $100-160 \mathrm{mM} \mathrm{KCl}, 20^{\circ} \mathrm{C}$. Excitation as described in the Experimental section. Excitation intensity: $2 \mu \mathrm{W}$.

A drastic increase in triplet amplitude of course has to be explained as all FCS measurements were taken at the same excitation beam power and a similar $\mathrm{pH}$-dependent effect to our knowledge has not been reported before. Both ISC yields as well as triplet relaxation rates have not yet been studied for protonated species of fluorescein. For fluorescein at $\mathrm{pH} 9$ the triplet relaxation rate has been determined to be $0.48 \times 10^{3} \mathrm{~s}^{-1}$ for free fluorescein. ${ }^{38}$ In our experiments we determined the triplet state relaxation rate of 5 -IAF at pH 13 to be $(0.56 \pm 0.04) \times 10^{3} \mathrm{~s}^{-1}$ (data not shown). Both $\mathrm{pH}$ values correspond to the dianion and are in good agreement considering that 5-IAF and fluorescein are not entirely identical. The importance of the influence of intersystem-crossing in FCS measurements has previously been reported. ${ }^{36,38}$

\subsection{Simulation guided analysis of experimental FCS data from probes at the surface of $\mathrm{CcO}$ from $P$. denitrificans}

In Section 3.3 we have shown that the increasing values of $g_{0}{ }^{-1}$ enable us to distinguish between overlapping reaction rates of singlet-triplet and protonation transitions. Using this strategy, 
$\mathrm{pH}$

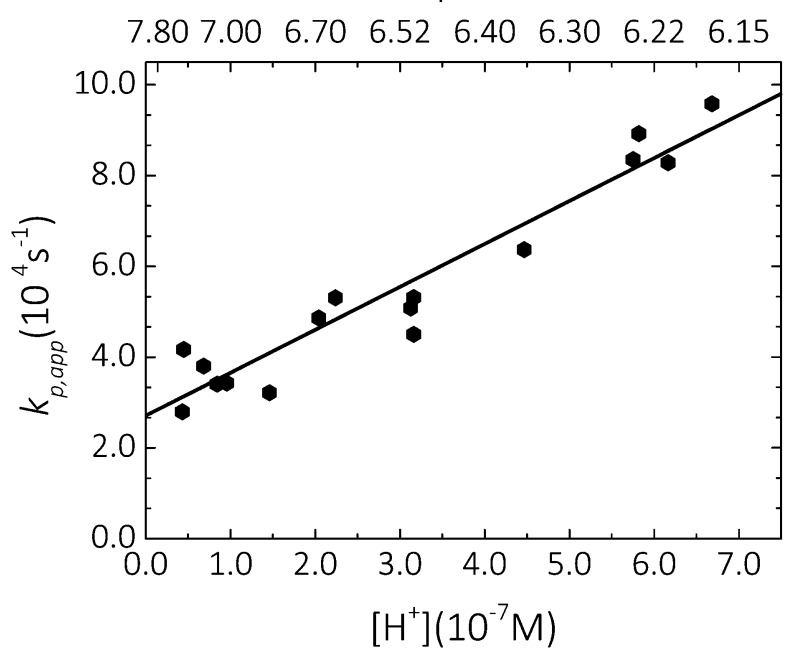

Fig. 6 Protonation rate $k_{\mathrm{p} \text {,app }}$ as a function of proton concentration for CcO CS-K299C-AF. The data were fitted to eqn (7). Conditions as in Fig. 5.

we obtained precise fluorescein protonation time constants allowing for improved reaction rate determination as will be shown below for fluorescein bound to the surface of $\mathrm{CcO}$.

Here, we have used the cysteine mutant CS-K299C to attach the fluorescein at the surface of $\mathrm{CcO}$ close to the entrance of the $\mathrm{K}$-channel. The protonation rate constants were plotted as a function of $\mathrm{pH}$ in Fig. 6. The linear $\mathrm{pH}$ dependence of the apparent protonation rates was fitted with eqn (7) and the corresponding protonation on and off rates were determined to $k_{+1}=(9.5 \pm 0.7) \times 10^{10} \mathrm{M}^{-1} \mathrm{~s}^{-1}$ and $k_{-1}=(2.7 \pm 0.3) \times 10^{4} \mathrm{~s}^{-1}$. The errors obtained in the simulation guided analysis for the on- and off-rates are $7.4 \%$ and $11.1 \%$, respectively. The on-rates determined at the two positions (301 and 299, Scheme 1b) close to the entrance of the K-channel in the oxidized state of the enzyme are quite similar with about $1 \times 10^{11} \mathrm{M}^{-1} \mathrm{~s}^{-1}$. We note that these rates are larger than those determined for free fluorescein in solution $\left(k_{+1}=4.0 \times 10^{10} \mathrm{M}^{-1} \mathrm{~s}^{-1}\right)$ and a literature value for a labeling position at the $\mathrm{CcO}$ surface $\left(k_{+1}=(7.1 \pm 0.8) \times 10^{10} \mathrm{~s}^{-1} \mathrm{M}^{-1}\right)$ in subunit III about $30 \AA$ away from the nearest proton input channel. ${ }^{17}$

However, these slightly increased protonation association rates in the oxidized state of the enzyme close to the K-channel do not explain the accelerated protonation time constants at the $\mathrm{N}$-side as observed in time-resolved flash-photolysis experiments of the $\mathrm{O} \rightarrow$ E step of the catalytic cycle. ${ }^{6}$ The absence of a dramatic increase in the protonation on rate (expected to be about $\left.10^{12}-10^{13} \mathrm{M}^{-1} \mathrm{~s}^{-117,20,37}\right)$ of the surface-attached probes close to K-channel (position 299 and 301) rules out a dramatic effect of a proton-collecting antenna close to these labeling sites. Scheme $1 \mathrm{~b}$ shows that position 299 is clearly surrounded by acidic residues and histidines, the constituents of a protoncollecting antenna. ${ }^{39}$ There are several possible explanations for the absence of highly accelerated protonation rates in our experiments. First, the acidic residues and histidines surrounding position 299 do not belong to a proton collecting antenna leading to the K-channel, or, second, the proton collecting properties are transiently turned on as suggested in our previous protonation experiments ${ }^{6}$ and the observed elevated rates in the initial oxidized state of $\mathrm{CcO}$ can be regarded as a pre-state of an proton collecting antenna. The former explanation fits with results from protonation dynamics experiments at the surface of $\mathrm{CcO}$ from $R$. sphaeroides, where the authors discussed the difference in density of acidic residues and histidines between the D- and K-channel and concluded that the entrance of the K-pathway appears not to provide an ideally optimized structure for proton collection. ${ }^{40}$ The interplay between protein surface properties and the properties of the membrane protein surrounding lipids contributing to accelerated protonation rates is also not clear. In our opinion, the formation of a proton collecting antenna around a proton channel entrance would rather depend on the properties of the protein surface itself. Dramatic increased protonation rate constants in several FCS experiments, however, were only observed as a function of the proton collecting antenna properties of lipid membranes. ${ }^{17,20,37}$

\section{Outlook}

In this report we determined precise local protonation rate constants at the entrance of the K-channel of oxidized $\mathrm{CcO}$. To selectively determine the contribution of the protein surface to the putative proton collective antenna we performed the experiments with solubilized $\mathrm{CcO}$. Our simulation guided analysis of experimental FCS data clearly improved the determination of protonation reaction rates from FCS curves. Whether the slightly increased protonation association rates close to the K-channel entrance are mechanistically relevant for proton uptake and electron-coupled proton pumping in $\mathrm{C} c \mathrm{O}$ will be determined in future studies of protonation rates of surfaceattached probes close to the $\mathrm{K}$ - and D-channel and in the different steps of the catalytic cycle. We expect that our simulation guided FCS analysis method allows to determine even small protonation rate changes as a function of the attachment position of the protonation probe at the protein or membrane surface. Furthermore, since singlet-triplet transitions are a common property of fluorescent dyes, our FCS analysis method may be of use in the treatment of other chemical processes leading to fluorescence fluctuations.

\section{Acknowledgements}

This project was supported by the Deutsche Forschungsgemeinschaft through grants to UA (Collaborative Research Center 1078, project A2 and Collaborative Research Center 1112, project B03).

\section{Notes and references}

1 H. Michel, Biochemistry, 1999, 38, 15129-15140.

2 L. Salomonsson, A. Lee, R. B. Gennis and P. Brzezinski, Biochim. Biophys. Acta, Bioenerg., 2004, 1658, 152. 
3 V. Sharma, G. Enkavi, I. Vattulainen, T. Rog and M. Wikström, Proc. Natl. Acad. Sci. U. S. A., 2015, 112, 2040-2045.

4 S. Iwata, C. Ostermeier, B. Ludwig and H. Michel, Nature, 1995, 376, 660-669.

5 S. Yoshikawa, K. Muramoto and K. Shinzawa-Itoh, Annu. Rev. Biophys., 2011, 40, 205-223.

6 K. Kirchberg, H. Michel and U. Alexiev, J. Biol. Chem., 2012, 287, 8187-8193.

7 Y. Marantz, E. Nachliel, A. Aagaard, P. Brzezinski and M. Gutman, Proc. Natl. Acad. Sci. U. S. A., 1998, 95, 8590-8595.

8 J. Riesle, D. Oesterhelt, N. A. Dencher and J. Heberle, Biochemistry, 1996, 35, 6635-6643.

9 A. Shinobu, G. J. Palm, A. J. Schierbeek and N. Agmon, J. Am. Chem. Soc., 2010, 132, 11093-11102.

10 U. Alexiev, P. Scherrer, T. Marti, H. G. Khorana and M. P. Heyn, FEBS Lett., 1995, 373, 81-84.

11 S. Dickopf, U. Alexiev, M. P. Krebs, H. Otto, R. Mollaaghababa, H. G. Khorana and M. P. Heyn, Proc. Natl. Acad. Sci. U. S. A., 1995, 92, 11519-11523.

12 U. Alexiev, T. Marti, M. P. Heyn, H. G. Khorana and P. Scherrer, Biochemistry, 1994, 33, 13693-13699.

13 U. Alexiev, R. Mollaaghababa, P. Scherrer, H. G. Khorana and M. P. Heyn, Proc. Natl. Acad. Sci. U. S. A., 1995, 92, 372-376.

14 J. Heberle, J. Riesle, G. Thiedemann, D. Oesterhelt and N. A. Dencher, Nature, 1994, 370, 379-382.

15 U. Alexiev, R. Mollaaghababa, H. G. Khorana and M. P. Heyn, J. Biol. Chem., 2000, 275, 13431-13440.

16 A. Springer, V. Hagen, D. A. Cherepanov, Y. N. Antonenko and P. Pohl, Proc. Natl. Acad. Sci. U. S. A., 2011, 108, 14461-14466.

17 L. Öjemyr, T. Sandén, J. Widengren and P. Brzezinski, Biochemistry, 2009, 48, 2173-2179.

18 Y. Marantz, O. Einarsdóttir, E. Nachliel and M. Gutman, Biochemistry, 2001, 40, 15086-15097.

19 Y. Georgievskii, E. S. Medvedev and A. A. Stuchebrukhov, Biophys. J., 2002, 82, 2833-2846.

20 T. Sandén, L. Salomonsson, P. Brzezinski and J. Widengren, Proc. Natl. Acad. Sci. U. S. A., 2010, 107, 4129-4134.
21 D. Magde, W. W. Webb and E. Elson, Phys. Rev. Lett., 1972, 29, 705-708.

22 Z. Petrasek and P. Schwille, J. R. Soc., Interface, 2009, 6, S15-S25.

23 U. Haupts, S. Maiti, P. Schwille and W. W. Webb, Proc. Natl. Acad. Sci. U. S. A., 1998, 95, 13573-13578.

24 K. Kirchberg, H. Michel and U. Alexiev, Biochim. Biophys. Acta, Bioenerg., 2013, 1827, 276-284.

25 R. Sjoback, J. Nygren and M. Kubista, Spectrochim. Acta, Part A, 1995, 51, L7-L21.

26 G. Kleymann, C. Ostermeier, B. Ludwig, A. Skerra and H. Michel, Bio/Technology, 1995, 13, 155-160.

27 B. Ludwig, Methods Enzymol., 1986, 126, 153-159.

28 B. Ludwig and G. Schatz, Proc. Natl. Acad. Sci. U. S. A., 1980, 77, 196-200.

29 H. Witt, V. Zickermann and B. Ludwig, Biochim. Biophys. Acta, 1995, 1230, 74-76.

30 M. Moller and U. Alexiev, Photochem. Photobiol., 2009, 85, 501-508.

31 A. Boreham, T. Y. Kim, V. Spahn, C. Stein, L. Mundhenk, A. D. Gruber, R. Haag, P. Welker, K. Licha and U. Alexiev, ACS Med. Chem. Lett., 2011, 2, 724-728.

32 S. R. Aragon and R. Pecora, J. Chem. Phys., 1976, 64, 1791-1803. 33 S. Ivanchenko and D. Lamb, in Supramolecular Structure and Function 10, ed. J. Brnjas-Kraljević and G. Pifat-Mrzljak, Springer, Netherlands, 2011, ch. 1, pp. 1-30.

34 C. T. Culbertson, S. C. Jacobson and J. M. Ramsey, Talanta, 2002, 56, 365-373.

35 U. Alexiev, T. Marti, M. P. Heyn, H. G. Khorana and P. Scherrer, Biochemistry, 1994, 33, 298-306.

36 J. Enderlein, G. I. D. Patra and J. Fitter, Curr. Pharm. Biotechnol., 2004, 5, 155-161.

37 M. Brändén, T. Sandén, P. Brzezinski and J. Widengren, Proc. Natl. Acad. Sci. U. S. A., 2006, 103, 19766-19770.

38 J. Widengren, U. Mets and R. Rigler, J. Phys. Chem., 1995, 99, 13368-13379.

39 G. Brändén, R. B. Gennis and P. Brzezinski, Biochim. Biophys. Acta, Bioenerg., 2006, 1757, 1052-1063.

40 M. Karpefors, P. Ädelroth, A. Aagaard, H. Sigurdson, M. S. Ek and P. Brzezinski, Biochim. Biophys. Acta, Bioenerg., 1998, 1365, 159-169. 